\title{
Influência da secagem convectiva sobre os compostos bioativos, estruturas morfológicas e perfil espectroscópico da polpa de muruci (Byrsonima crassifolia)
}

\author{
Influence of convective drying on bioactive compounds, morphological structures and \\ spectroscopic profile of muruci pulp (Byrsonima crassifolia) \\ O. V. Santos ${ }^{1 *}$; J. L. S. B. Cardoso ${ }^{1}$; S. D. Soares ${ }^{2}$; M. G. Martins²; F. C. A. \\ Nascimento $^{1}$; B. E. Teixeira-Costa ${ }^{3}$ \\ ${ }_{1}^{1}$ Instituto de Ciências da Saúde/Faculdade de Nutrição, Universidade Federal do Pará, 66071-110, Belém-Pará, \\ Brasil \\ ${ }^{2}$ Instituto de Tecnologia/Programa de Pós-graduação em Ciência e Tecnologia de Alimentos, Universidade Federal \\ do Pará, 66071-110, Belém-Pará, Brasil \\ ${ }^{3}$ Departamento de Engenharia de Alimentos/ Faculdade de Ciências Agrárias, Universidade Federal do Amazonas, \\ 69077-000 Manaus-Amazonas, Brasil
}

orquideavs@ufpa.br

(Recebido em 18 de agosto de 2020; aceito em 12 de novembro de 2020)

\begin{abstract}
O muruci (Byrsonima crassifolia) é um fruto comumente consumido na forma de polpas que precisa de aplicação de técnicas de secagem viáveis economicamente para prolongar o consumo nos períodos de entressafra. Nesse sentido, o objetivo desta pesquisa foi analisar a influência da secagem convectiva sobre os compostos bioativos, estruturas morfológicas e perfil espectroscópico da polpa de muruci. As normas metodológicas seguiram os padrões da Association of Official Analytical Chemists e de publicações aceitas internacionalmente. Os dados mostram uma matéria-prima ácida com elevado conteúdo em sólidos solúveis $\left(14,45^{\circ}\right.$ Brix $)$ e alta estabilidade pós secagem ( 0,28 de atividade de água). Em relação aos constituintes funcionais apresentou alto conteúdo em vitamina $C$ tanto in natura como em forma de pó (168,5 a 140,3 $\mathrm{mg} / 100 \mathrm{~g})$. Os teores em flavonoides tiveram decréscimo de $35 \%(47,55 \mathrm{mg} / 100 \mathrm{~g}$ para $35,2 \mathrm{mg} / 100 \mathrm{~g})$ do fruto in natura para a polpa seca, semelhante ao comportamento dos compostos fenólicos com redução média de 20\% (143,35 para 118,1 mgAGE/100g). Constatou-se altos teores de carotenoides (325,8 $\mu \mathrm{g} / 100 \mathrm{~g}$ de $\beta$-caroteno), cuja conversão para vitamina A condiz a aproximadamente $12 \%$ das recomendações diárias dessa vitamina. Os perfis espectroscópicos mostraram grupos químicos funcionais e não foram observados picos de compostos relacionados a processos de degradação oxidativa. A morfologia dos grânulos mostrase de forma cilíndrica de aspecto esponjoso tendo em seu interior estruturas arredondadas semelhantes ao amido. Esses dados evidenciam a potencialidade da conversão da polpa deste fruto na forma de pó agregando compostos bioativos com alta estabilidade e potencial fonte de vitamina $\mathrm{A}$.
\end{abstract}

Palavras-chave: Byrsonima crassifolia, pó, bioativos

Muruci (Byrsonima crassifolia) is a fruit commonly consumed in the form of pulps that needs the application of economically viable drying techniques to prolong consumption in the off-season. In this sense, the aim of this research was to analyze the influence of convective drying on the bioactive compounds, morphological structures and spectroscopic profile of the muruci pulp. Methodological standards followed the standards of the Association of Official Analytical Chemists and internationally accepted publications. The data show an acidic raw material with a high content of soluble solids (14.45 ${ }^{\circ}$ Brix) and high post-drying stability ( 0.28 of water activity). Regarding the functional constituents, it had a high content of vitamin C both in natural and in powder form (168.5 to $140.3 \mathrm{mg} / 100 \mathrm{~g})$. Flavonoid levels decreased by $35 \%$ ( $47.55 \mathrm{mg} / 100 \mathrm{~g}$ to $35.2 \mathrm{mg} / 100 \mathrm{~g}$ ) of fresh fruit for dry pulp, similar to the behavior of phenolic compounds with an average reduction of $20 \%$ (143.35 to 118, $1 \mathrm{mgAGE} / 100 \mathrm{~g})$. High levels of carotenoids ( $325.8 \mu \mathrm{g} / 100 \mathrm{~g}$ of $\beta$-carotene) were found, whose conversion to vitamin A corresponds to about $12 \%$ of the daily recommendations of this vitamin. The spectroscopic profiles showed functional chemical groups and peaks of compounds related to oxidative degradation processes were not observed. The granules morphology shows cylindrical shape with a spongy aspect, with rounded structures like starch inside. These data show the potential of the conversion of the pulp of this fruit into powder, adding bioactive compounds with high stability and potential source of vitamin A.

Keywords: Byrsonima crassifolia, powder, bioactive 


\section{INTRODUÇÃO}

A grande biodiversidade de frutos amazônicos com peculiaridade de sabores, cores, aromas e constituições nutricionais e bioativas, tais características habilitam esses frutos como incremento industrial caso as etapas de sua cadeia produtiva forem estendidas para além dos períodos de safras, com aplicações diversificadas como matéria-prima de base ou em misturas para o design de outros produtos. Neste contexto, o murucizeiro é uma árvore de pequeno a médio porte (podendo chegar até 6 metros), pertencente a família Malpighiaceae, que cresce principalmente em locais de clima tropical e terrenos secos, distribuindo-se em todas as regiões do Brasil. Os frutos dessa árvore são também conhecidos como murici-do-campo, murici-da-praia, nanche (em inglês) dentre outras denominações. Trata-se de uma fruta pequena medindo cerca de $5 \mathrm{~mm}$ de diâmetro, contendo apenas um caroço em seu interior (semente) e polpa (mesocarpo) pastosa de cor amarela quando madura, de cheiro e sabor marcantes. Seu consumo é primordialmente in natura na forma de polpa, assim sendo utilizada na produção de sucos, sorvetes, geleias, além de ser possível extrair óleo da semente que são aplicadas em preparações culinárias e na produção de cosméticos [1].

Pesquisas têm sido realizadas com esse fruto (in natura) para avaliar sua composição e atividade antioxidante [2], têm-se dado destaque a constituição de fibras, cujos teores de proteínas giram em torno de 5,45\%, seguidos de carboidratos e lipídios com predominância de ácidos graxos insaturados [3]. Também se evidencia o muruci como fonte de minerais, vitaminas e de compostos fenólicos (ácido gálico e quercetina) e carotenoides (luteínas e zeaxantinas), garantindo uma boa capacidade antioxidante [4].

A ênfase das pesquisas neste fruto recai sobre seu estado in natura, e/ou isolamento de partes, mas ainda não se avançou no sentido de suprir a necessidade da demanda durante a entressafra e poucas contribuições referente ao incremento de aplicações em outros produtos. A relação custobenefício quanto as aplicações de tecnologias de secagem podem tornar inviável a obtenção de produto como na forma de pó solubilizável para população comumente consumidora do fruto. $\mathrm{O}$ potencial econômico do muruci pode ser melhor explorado com aplicação de tecnologias que aumentem sua estabilidade, reduza os teores de umidade, principalmente nas condições edafoclimáticas típicas da região amazônica, para assim potencializar sua comercialização para diferentes partes do mundo, tal como ocorre com o açaí $[5,6]$.

Nesse sentido, alternativas como a aplicação de tecnologias, por exemplo, a secagem convectiva, pode ser utilizada, pois trata-se de uma das formas de incrementar sua cadeia produtiva, mantendo o fornecimento deste fruto nos períodos de entressafras. Tal processo habilita-se como método viável, simples e barato mantendo uma relação positiva entre o custo e o benefício [7]. Diante desse contexto, o objetivo desta pesquisa foi analisar a influência da secagem convectiva sobre os compostos bioativos, estruturas morfológicas e perfil espectroscópico da polpa de muruci (Byrsonima crassifolia).

\section{MATERIAL E MÉTODOS}

\subsection{Preparo da amostra}

As amostras de muruci foram obtidas no mercado do Ver-o-Peso localizado na região metropolitana do município de Belém, estado do Pará, Brasil. Em seguida foram transportadas em sacos plásticos de polietileno de baixa densidade (PEBD) e armazenadas no Laboratório de Ciência dos Alimentos da Universidade Federal do Pará (UFPA), à temperatura de $7{ }^{\circ} \mathrm{C}$. Na etapa seguinte foram realizados os procedimentos de seleção, lavagem, sanitização, despolpamento, secagem em estufa de circulação forçada (marca Thot, modelo 170) na temperatura de $50^{\circ} \mathrm{C}$ por $8 \mathrm{~h}$.

\subsection{Obtenção do extrato aquoso}


O extrato aquoso foi obtido segundo a metodologia de Vieira (2011) [8], com extração na proporção de 1:20 (amostra:solvente), utilizando como solvente a água destilada. A amostra foi homogeneizada durante 30 minutos em erlenmeyer no agitador magnético Fisatom, seguido de centrifugação a 3500 rpm em centrífuga LS-3 Plus CELM.

\subsection{Análises físicas}

A atividade de água do material foi obtida por meio de medida direta, em instrumento Labmaster-aw neo (series 3TE, marca Novasina), com controle interno de temperatura a $25{ }^{\circ} \mathrm{C}$;

$\mathrm{O}$ pH foi determinado seguindo o método de $\mathrm{n}^{\circ} 981.12$ da AOAC (2010) [9], medida em potenciômetro MS Tecnopon Mpa-210;

A acidez total titulável foi verificada de acordo com as normas expressas pelo Instituto Adolf Lutz (1985) [10], com resultados descritos em porcentagem (\%) de ácido cítrico;

Os sólidos solúveis totais (SST) foram obtidos por meio do índice de refração de acordo com o Instituto Adolf Lutz (2008) [11] através da leitura em refratômetro de bancada (marca Tecnal, modelo AR/200 digital), expresso em ${ }^{\circ}$ Brix.

\subsection{Análise da concentração de compostos bioativos}

\subsubsection{Carotenoides totais:}

A determinação do conteúdo total de carotenoides do muruci foi realizada por leitura espectroscópica em absorbância de $450 \mathrm{~nm}$, conforme as metodologias descritas por RodriguezAmaya (2001) [12] (Equação 1).

$$
\text { Carotenos }(p p m)=\frac{V(m L) \times A}{2592 \times m_{0}} 10^{6}
$$

Em que, $\mathrm{V}=$ Volume total $(\mathrm{mL})$

$\mathrm{m}_{0}=$ Massa da amostra $(\mathrm{g})$

A = Absorbância

\subsection{Análise de flavonoides e antocianinas}

Os flavonoides e antocianinas foram determinados de acordo com a metodologia descrita por Lees e Francis (1972) [13] com leitura em espectrofotômetro UV-Vis (modelo IL- 592, marca Kasuaki) no comprimento de onda de $374 \mathrm{~nm}$ para flavonoides e $535 \mathrm{~nm}$ para antocianinas.

\subsection{Polifenóis totais}

O teor de polifenóis totais foi analisado de acordo com o ensaio de Folin Ciocalteu, tal como descrito por Ali Akbarian, Casazza e Perego (2011) [14], utilizando-se um espectrofotômetro UVVis, modelo IL- 592 KASUAKI, a um comprimento de onda de $725 \mathrm{~nm}$ e os resultados foram calculados através da curva-padrão de ácido gálico com equação da reta y=0,0017x $\left(\mathrm{R}^{2}=0,9966\right)$.

\subsection{Espectroscopia infravermelha (FTIR)}

A análise de espectroscopia de infravermelho com transformada de Fourier (FTIR) foi realizada usando um espectrômetro Perkin Elmer, modelo Frontier 98737 (Waltham, MA, EUA) a $25^{\circ} \mathrm{C}$ na faixa de $4000-400 \mathrm{~cm}^{-1}$. Os espectros da amostra foram registrados calculando a média de 20 varreduras com resolução de $4 \mathrm{~cm}^{-1}$ no modo de transmissão. 


\subsection{Análise morfológica}

As análises morfológicas dos grânulos foram realizadas pela visualização em microscopia eletrônica de varredura (MEV), com amostras acopladas em suportes e metalizados com $\mathrm{Au} / \mathrm{Pd}$ para permitir condutividade elétrica necessária no processo de formação das imagens, em corrente do feixe de elétrons de 85-90 $\mu \mathrm{A}$. As imagens ou eletromicrografias foram realizadas em microscópio eletrônico de varredura marca Tescan, modelo Vega3, instalado no Laboratório de Nanomanipulação do Programa de Pós-Graduação em Física (PPGF/ UFPA).

\section{RESULTADOS}

\subsection{Características físico-químicas}

Na Tabela 1 são apresentadas as características físico-químicas da polpa in natura e em pó de muruci.

Tabela 1: Características físico-químico da polpa in natura e em pó de muruci.

\begin{tabular}{crccc}
\hline Parâmetros & \multicolumn{1}{c}{$\begin{array}{c}\text { Polpa } \\
\text { in natura }\end{array}$} & $\begin{array}{c}\text { Polpa } \\
\text { Seca }\end{array}$ & $\begin{array}{c}\text { López et al. } \\
(\mathbf{2 0 1 4})[\mathbf{1 8}]\end{array}$ & $\begin{array}{c}\text { Alves et al. } \\
(\mathbf{2 0 2 0})[\mathbf{1 9}]\end{array}$ \\
\hline pH & $3,80 \pm 0,02$ & $3,35 \pm 0,05$ & - & - \\
Sólidos solúveis ( ${ }^{\circ}$ Brix) & $15,75 \pm 0,10$ & $14,45 \pm 0,45$ & $0,00 \pm 1,00$ & 5,56 \\
Atividade de água (Aw) & $0,92 \pm 0,05$ & $0,28 \pm 0,04$ & $0,359 \pm 0,03$ & 0,98 \\
$\begin{array}{c}\text { Acidez titulável } \\
\text { (\% ácido cítrico) }\end{array}$ & $2,95 \pm 0,25$ & $2,30 \pm 0,25$ & $3,60 \pm 0,17$ & - \\
\hline
\end{tabular}

*Dados representam média \pm desvio padrão das triplicatas.

No presente estudo o pH apresentou médias de 3,8 a 3,35 ácido em ambas as formas pesquisadas, semelhante aos dados encontrados no estudo de Carvalho e do Nascimento (2016) [15], no qual a média dos valores de pH variaram entre 3,10 e 3,90 nos genótipos estudados. Monteiro e Pires (2016) [16] e da Conceição Souza et al. (2019) [17] apresentaram valores de pH semelhantes entre si (3,20 e 3,22 respectivamente) e ligeiramente menores aos encontrados na presente pesquisa.

Os sólidos solúveis totais apresentaram médias de 15,75 e 14,45 ( ${ }^{\circ}$ Brix) entre o valor in natura e o seco em pó, mostrando-se próximos aos resultados de López et al. (2014) [18] com frutos in natura e superiores aos apresentados nas pesquisas de Alves et al. (2020) [19] mostrados na tabela 1. De acordo com a Instrução Normativa $n^{\circ} 37$, de $1^{\circ}$ de outubro de 2018 e Portaria $n^{\circ} 58$, de 30 de agosto de 2016 (Brasil, 2016 e 2018) [20,21] o valor mínimo de sólidos solúveis totais é de $4,4{ }^{\circ}$ Brix, sendo perceptível que os resultados da presente pesquisa estão de acordo com a legislação vigente.

A atividade de água verificada no estudo apresentou média de 0,92 na polpa in natura com redução para 0,28 na polpa seca. Houve aumento da estabilidade deste material como resultado da aplicação da secagem convectiva que ao se comparar com as pesquisas de Alves et al. (2020) [19] em amostras in natura com 0,98 de atividade de água (tanto na casca quanto polpa) mostra essa diferença de estabilidade. Em López et al. (2014) [18] com muruci liofilizado $(0,243)$ e desidratado $(0,359)$ os valores de atividade de água foram semelhantes aos desta pesquisa. Esta variável mostra a estabilidade e a não perecibilidade deste produto, com boa vida de prateleira, por conta da redução da proliferação de microrganismos [22-27]. É importante enfatizar que essa diminuição de atividade de água está relacionada com o método de secagem utilizado. A secagem convectiva é um método de desidratação acessível economicamente que tem como um de seus objetivos aumentar a vida de prateleira e manter as características sensoriais e nutricionais [27].

Já a acidez total titulável encontrada no estudo apresentou médias de 2,95 in natura com redução para 2,30 (\% ácido cítrico) no material seco, próximo ao encontrado por Mota et al. 
(2016) [24] que demonstraram valores entre 1,41 e 2,93 nos frutos armazenados, superior aos achados de Peralta et al. (2020) [25] nos frutos secos por três dias a temperatura de $70{ }^{\circ} \mathrm{C}(0,64 \%)$.

A partir desses dados comparativos é possível confirmar que a polpa seca por convecção de muruci é ácida, bem como sua forma in natura a qual também apresenta baixo $\mathrm{pH}$ e, consequentemente, elevada acidez $[18,24,28]$. Tais análises possuem relevância significativa, pois as mesmas estão diretamente ligadas ao desenvolvimento industrial visto que há maior estabilidade microbiológica, rendimento dos produtos quando realizada diluições e ausência da necessidade da etapa de adição de acidulantes [29, 30].

\subsection{Compostos bioativos}

Os teores totais de carotenoides estão apresentados na Tabela 2 expressos em $\beta$-caroteno e convertido em vitamina A. Esta conversão considera que $1 \mu \mathrm{g}$ de $\beta$-caroteno é equivalente a 0,167 $\mu \mathrm{g}$ de retinol (RE) determinado pela Resolução da Diretoria Colegiada RDC no 269 de 22 de setembro de 2005 [29]. Os dados desta pesquisa foram comparados a ingestão diária recomendada (IDR) para diferentes faixas etárias da população brasileira [29].

Tabela 2: Carotenoides totais, convertidos em Equivalente de Retinol (RE).

\begin{tabular}{|c|c|c|c|c|}
\hline $\begin{array}{c}\text { Polpa em pó } \\
\beta \text {-caroteno } \\
(\mu \mathrm{g} / 100 \mathrm{~g})\end{array}$ & $\begin{array}{c}\mathrm{RE} \\
\mu \mathrm{g} / \mathbf{1 0 0 g})\end{array}$ & $\begin{array}{c}\text { Polpa in natura } \\
\beta \text {-caroteno } \\
(\mu \mathrm{g} / \mathbf{1 0 0 g})\end{array}$ & $\begin{array}{c}\mathbf{R E} \\
(\mu \mathrm{g} / 100 \mathrm{~g})\end{array}$ & $\begin{array}{l}\text { IDR Vitamina A } \\
\text { (Brasil, 2005) [29] }\end{array}$ \\
\hline $325,8 \pm 3,55$ & 54,40 & $112,0 \pm 3,50$ & 18,70 & $\begin{array}{c}\text { Criança 1-3 anos: } 400 \mu \mathrm{g} \mathrm{RE} \\
\text { Criança 4-6 anos: } 450 \mu \mathrm{g} \mathrm{RE} \\
\text { Criança 7-10 anos: } 500 \mu \mathrm{g} \mathrm{RE} \\
\text { Adultos: } 600 \mu \mathrm{g} \mathrm{RE} \\
\text { Gestantes: } 800 \mu \mathrm{g} \mathrm{RE} \\
\text { Lactantes: } 850 \mu \mathrm{g} \mathrm{RE}\end{array}$ \\
\hline
\end{tabular}

*Médias \pm desvio padrão das triplicatas.

A média dos teores de carotenoides foi de 325,8 $(\mu \mathrm{g} / 100 \mathrm{~g})$ na polpa em pó e de $112(\mu \mathrm{g} / 100 \mathrm{~g})$ in natura, próximas aos valores encontrados por Belisário et al. (2020) [30] em amostra in natura de muruci no primeiro dia de colheita $(100,0 \pm 5,3 \mu \mathrm{g} / 100 \mathrm{~g})$, nas pesquisas de Franklin e Nascimento (2020) [31] (fruta com casca - 570,00 $\mu \mathrm{g}$ de $\beta$-caroteno e sem casca - $66 \mu \mathrm{g}$ de $\beta$ caroteno), na primeira situação encontraram valores superiores aos do presente estudo e na segunda, percebe-se drástica redução da quantidade de $\beta$-caroteno.

Em comparação com frutos descritos como fontes de $\beta$-caroteno na Tabela Brasileira de Composição de Alimentos [32] em estado in natura, o muruci ultrapassa valores de frutas como a laranja (RE variando entre 2 e $4 \mu \mathrm{g} / 100 \mathrm{~g}$ de acordo a variação da fruta) e tangerina $(\mathrm{RE}=49 \mu \mathrm{g} / 100 \mathrm{~g})$ e apresenta valores menores ao ser comparado com acerola ( $\mathrm{RE}=60 \mu \mathrm{g} / 100 \mathrm{~g})$, mamão (RE variando entre 77, 78 e $93 \mu \mathrm{g} / 100 \mathrm{~g}$ de acordo a variação da fruta), maracujá (RE variando entre 78 e $138 \mu \mathrm{g} / 100 \mathrm{~g}$ de acordo a variação da fruta), pitanga (RE=164 $\mu \mathrm{g} / 100 \mathrm{~g})$, cajá $(\mathrm{RE}=167 \mu \mathrm{g} / 100 \mathrm{~g})$, manga (RE variando entre 100,113 e $793 \mu \mathrm{g} / 100 \mathrm{~g}$ de acordo a variação da fruta) e nêspera (RE podendo chegar até $306 \mu \mathrm{g} / 100 \mathrm{~g}$ de acordo a variação da fruta).

Nas pesquisas de Britton e Khachik (2009) [33] há a proposição de uma classificação para quantidade de carotenoides em alimentos: baixa (0-100 $\mu \mathrm{g} / 100 \mathrm{~g})$, moderada (100-500 $\mu \mathrm{g} / 100 \mathrm{~g})$, alta $(500-2000 \mu \mathrm{g} / 100 \mathrm{~g})$ e muito alta $(\geq 2000 \mu \mathrm{g} / 100 \mathrm{~g})$. Com base nesta classificação a polpa de muruci in natura e seca por convecção expressou moderada concentração de carotenoides.

O consumo deste composto está associado a diversos benefícios, dentre eles a redução do risco de Doenças Crônicas Não Transmissíveis (DCNT) [34, 35]. Observa-se nos resultados referencias de IDR de vitamina A na Tabela 2 que o consumo de $100 \mathrm{~g}$ do fruto em pó de muruci atende em torno de $12 \%$ da recomendação diária desse micronutriente regulador orgânico.

$\mathrm{O} B$-caroteno na sua fração mais bioativa apresenta função de provitamina A no organismo humano, com $100 \%$ de atividade [36, 37]. Tal vitamina está relacionada a regulação da visão, 
reprodução, redução da gordura corporal (por ação na expressão genética, impedindo a formação de tecido adiposo por meio do bloqueio da diferenciação celular), síntese de progesterona, redução do risco de doenças crônicas como diabetes tipo 1 e cânceres como de próstata, mama, pulmão, bexiga, cervical e gástrico [38-41].

A Tabela 3 apresenta outros compostos bioativos contidos na polpa muruci in natura e em pó.

Tabela 3: Determinação de compostos bioativos na polpa in natura e em pó seca de murici.

\begin{tabular}{ccc}
\hline Parâmetros & Polpa in natura & Polpa em pó \\
\hline Compostos fenólicos totais (mg AGE/100g) & $143,35 \pm 5,2$ & $118,15 \pm 3,5$ \\
Flavonoides (mg/ 100g) & $47,55 \pm 1,51$ & $35,2 \pm 0,55$ \\
Vitamina C (mg/ 100g) & $108,5 \pm 1,55$ & $140,3 \pm 1,35$ \\
\hline
\end{tabular}

*Médias \pm desvio padrão das triplicatas.

Os resultados dos compostos fenólicos ficaram entre 118,15 a 143,35 (mg AGE/100g) apresentando valores superiores aos encontrados por Alves et al. (2020) [19] (extrato etéreo: 0,04 \pm 0,01 e extrato aquoso: $0,22 \pm 0,01 \mathrm{mg} \mathrm{AGE} / 100 \mathrm{~g}$ ) em amostras da fruta in natura e inferiores se comparado a outros frutos da Amazônia como o tucumã (Astrocaryum vulgare Mart) 159 (mg AGE/100g b.s.), bacuri (Platonia insignis) 521 (mg AGE/100g) e o cupuaçu (Theobroma grandiflorum) 305 (mg AGE/100g) [42, 43].

Em relação aos teores de flavonoides comparado com as pesquisas de Gonçalves et al. (2017) [42], com amostras de murici-pitanga em diversos estágios de maturação, percebe-se que o fruto maduro $(14,59 \mathrm{mg} / 100 \mathrm{~g})$ apresenta resultados inferiores aos da presente pesquisa. Em comparação com Montenegro et al. (2017) [43] averígua-se valores semelhantes de flavonoides presentes no muruci in natura $(49,41 \pm 3,45$ e $45,20 \pm 9,40 \mu \mathrm{g} / \mathrm{g})$. Este constituinte atua conjuntamente com outros antioxidantes como as vitaminas $\mathrm{C}$ e $\mathrm{E}$. O consumo de alimentos contendo flavonoides como o muruci relaciona-se a prevenção de diversos tipos de canceres, bem como na redução da glicemia, colesterol, níveis pressóricos e triglicerídeos.

A vitamina C, segundo Andrade et al. (2002) [44] pode ser classificada levando em consideração diferentes teores: fontes elevadas (100 a $300 \mathrm{mg} / 100 \mathrm{~g}$ ), fontes médias (50 a 100 $\mathrm{mg} / 100 \mathrm{~g}$ ) e fontes baixas $(25 \mathrm{a} 50 \mathrm{mg} / 100 \mathrm{~g}$ ), portanto é possivel afirmar que o muruci possui elevadas quantidades de tal composto. Os valores encontrados na atual pesquisa para a fruta ultrapassam os achados de Carvalho e do Nascimento (2016) [15] em base úmida (variando entre $16,81 \pm 0,55$ e $86,64 \pm 0,01 \mathrm{mg} / 100 \mathrm{~g}$ nos genótipos estudados) e de Moreira-Araújo et al. (2019) [4] em polpa fresca $(58,60 \pm 1,32 \mathrm{mg} / 100 \mathrm{~g})$. Além disso, o murici apresenta valores superiores a diversas fruta amazônicas, como abiu $(5,34 \pm 0,44 \mathrm{mg} / 100 \mathrm{~g})$, bacuri $(28,31 \pm 3,23 \mathrm{mg} / 100 \mathrm{~g})$, biribá $(10,00 \pm 0,13 \mathrm{mg} / 100 \mathrm{~g})$, cupuaçu $(52,59 \pm 0,33 \mathrm{mg} / 100 \mathrm{~g})$, uxi $(15,04 \pm 2,03 \mathrm{mg} / 100 \mathrm{~g})$, monguba $(5,20 \pm 0,16 \mathrm{mg} / 100 \mathrm{~g})$ e pajurá $(7,72 \pm 0,69 \mathrm{mg} / 100 \mathrm{~g})[40]$

O mesmo é percebido ao comparar as amostras desta pesquisa com outras conhecidas como ricas em vitamina $\mathrm{C}$ a exemplo da mexerica $(111,97 \mathrm{mg} / 100 \mathrm{~g}$ ), goiaba (variando entre $97,7 \mathrm{e}$ $89,08 \mathrm{mg} / 100 \mathrm{~g}$ de acordo com o tipo), kiwi $(70,78 \mathrm{mg} / 100 \mathrm{~g})$, morango $(69,80 \mathrm{mg} / 100 \mathrm{~g})$, manga (65,52 mg/100g), laranja (47,32 mg/100g), açaí (42,00 mg/100g) e limão (32,78 mg/100g) [32].

Ao fazer tais comparações, é importante destacar que dependendo do processo de secagem (além de outros fatores como tempo e temperatura) podem haver modificações no conteúdo de nutrientes, antioxidantes e compostos bioativos (carotenoides, compostos fenólicos, vitaminas etc.), levando ao aumento ou redução dos mesmos [45]. A vitamina C, por exemplo, é sensível a diversos fatores enzimáticos e físicos, como é o caso da temperatura que durante o processo de secagem pode facilmente levar a perda de tal composto; por outro lado, em algumas situações os teores de ácido ascórbico podem mostrar-se aumentados após o processo de secagem em função da inativação da enzima ascorbato oxidase [46].

A composição química desta matéria-prima é apresentada no espectro de bandas, frequência e grupos funcionais na Figura 1. 


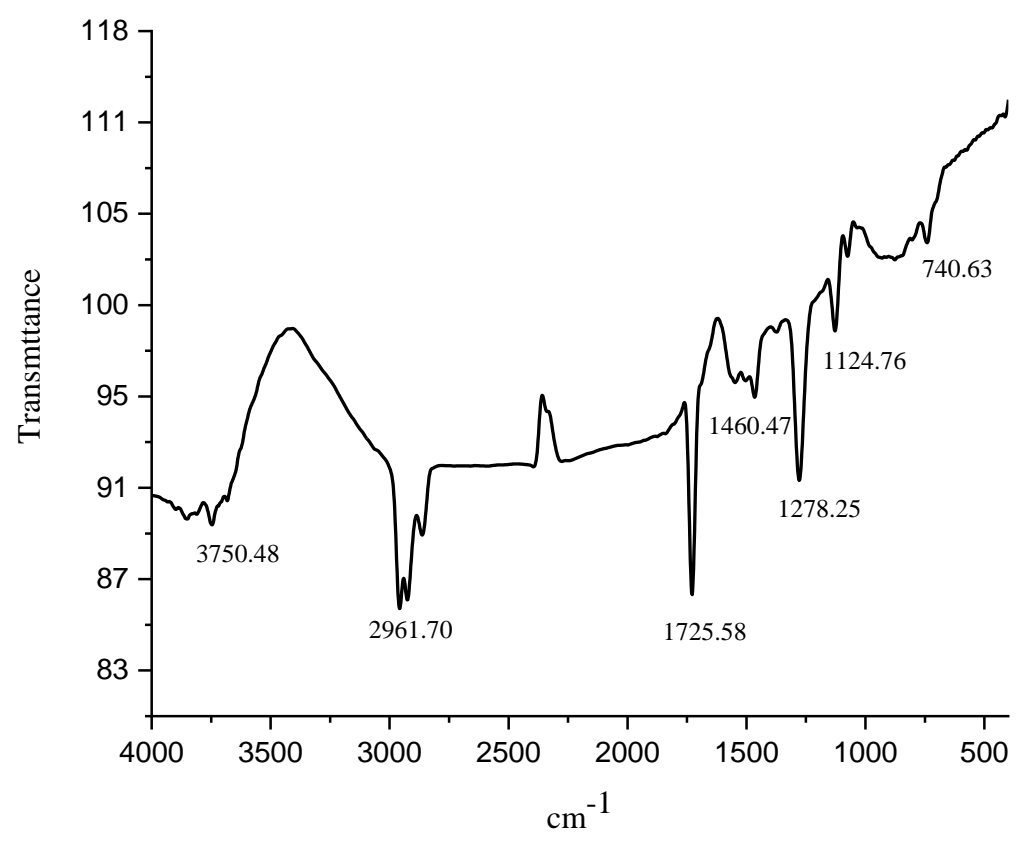

Figura 1: Espectroscopia da polpa de Muruci em pó.

O comportamento dos picos espectrais no material em pó apresenta perfis com picos que demostram sua constituição em compostos orgânicos. Os padrões espectrais evidenciam a presença de bandas em destaque abaixo de $3000 \mathrm{~cm}^{-1}$ até $1278 \mathrm{~cm}^{-1}$, característicos de compostos orgânicos como os álcoois, ésteres, éteres, ácidos carboxílicos. Intervalo de frequência em torno de $1725 \mathrm{~cm}^{-1}$ estão presentes e caracterizam compostos do grupo carbonila $(\mathrm{C}=\mathrm{O})$, metil ésteres, cetonas, aldeídos frequentes em materiais que contem perfil de ácidos graxos de cadeia longa. Dentre os principais constituintes químicos relacionados não se observa picos com forte intensidade que indiquem processos de degradação oxidativos nestes materiais [46-48].

Observa-se a presença da banda situada na faixa em torno de $1278 \mathrm{~cm}^{-1}$ a $1124 \mathrm{~cm}^{-1}$ característico do grupo funcional carbonila (C-O) encontrados em grupos químicos como os álcoois, ésteres, éteres, ácidos carboxílicos. As menores bandas verificadas estão em torno de 740 $\mathrm{cm}^{-1}$, outras bandas relacionadas à cadeia alifática de ácidos graxos podem estar ligadas à sequência de cadeias e anéis aromáticos de ácidos graxos [46, 48].

\subsection{Análise morfológica}

Outro aspecto relevante analisado refere-se às estruturas morfológicas dessa matéria-prima após o processo de secagem convectiva, cujas estruturas e macrocomponentes estão apresentados nas Figuras 2 e 3. 


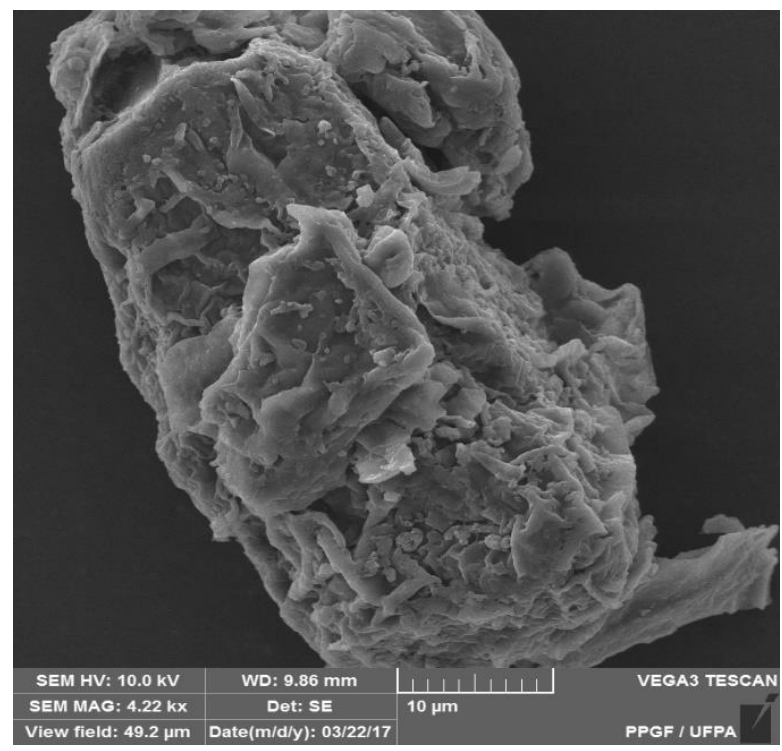

Figura 2: Grânulo de formato e superfície irregular.

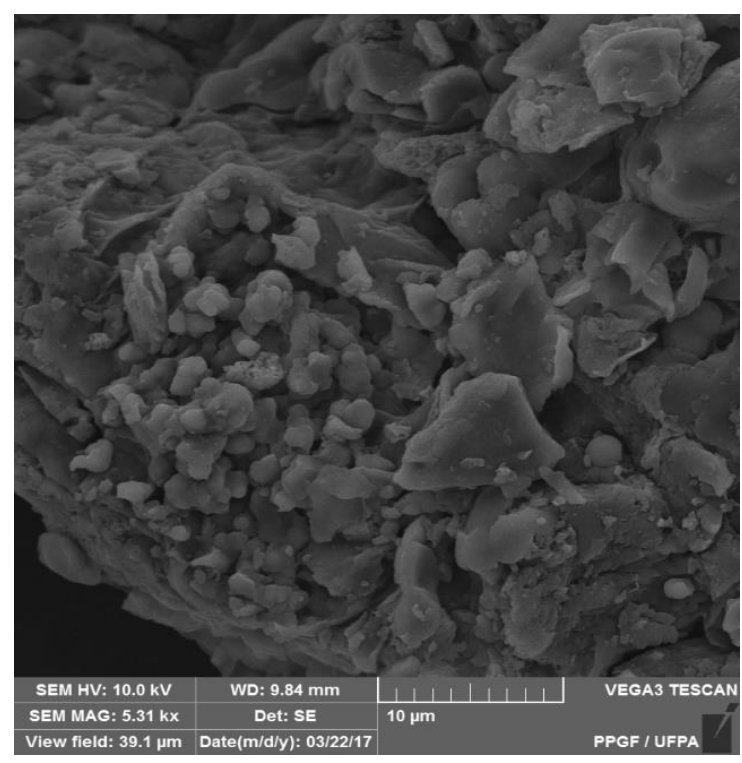

Figura 3: Superfície externa do grânulo em destaque.

A morfologia da polpa seca em pó de muruci é mostrada de forma ampla na Figura (2). A estrutura de seus grânulos tem formato próximo ao cilíndrico, com superfície irregular com pequenas reentrâncias envolto em estruturas residuais de parênquima vegetal fraturados, provavelmente como resultado do processo de despolpamento e secagem.

Na Figura 3 são apresentadas as estruturas ampliadas em destaque, com superfície externa dos grânulos contendo involuções com estruturas em forma arredondada semelhantes a amidos, com mais espaços e reentrâncias em seu formato mantem-se irregular.

As estruturais morfológicas mostram superfícies externas (figura 3) com aspecto irregular que podem ser considerados benéficos a aplicação alimentar dessa matéria-prima. Como a capacidade de absorver e reter água e outros solventes não polares, como os lipídios, pois essas características podem promover sua utilização em segmentos de panificação e massas em geral, aliando suas propriedades bioativas e tecnológicas, sendo assim aplicáveis a diversos segmentos industriais. 


\section{CONCLUSÃO}

A aplicação de processos de secagem convectiva na polpa dos frutos do muruci mostra-se uma tecnologia viável no prolongamento de sua cadeia produtiva nos períodos de entressafra. $\mathrm{Na}$ forma de pó esse fruto apresenta alta estabilidade microbiológica, mantendo sua acidez comparado com a polpa in natura.

A composição funcional tanto das polpas in natura quanto na forma de pó do muruci revela que ambas são fontes de bioativos, com destaque para o incremento apresentado no teor de carotenoides na forma de pó, sua funcionalidade observada na conversão em teores de vitamina A suprem teores relevantes da IDR.

O perfil espectroscópico desse fruto mostra a presença de grupos funcionais relacionados a grupos cetônicos, amidas relacionadas as proteínas, grupos aldeídos e metil ésteres característicos de ácidos graxos de cadeia longa, como os insaturados. Outro dado em seu perfil a ser destacado é a ausência de grupos químicos relacionados a processos de oxidação.

$\mathrm{Na}$ análise morfológica dos grânulos desse pó evidenciou-se uma estrutura cilíndrica com superfície que apresentam reentrâncias e presença de amidos, característica que podem potencializar sua capacidade de reidratação e aplicações em diversos segmentos industriais.

\section{AGRADECIMENTOS}

Os autores agradecem ao LABNANO-AMAZON/UFPA pelo apoio através das instalações de MEV utilizadas no presente trabalho.

\section{REFERÊNCIAS BIBLIOGRÁFICAS}

1. Coradin L, Camillo J, Pareyn FGC. Espécies nativas da flora brasileira de valor econômico atual ou potencial: plantas para o futuro: região Nordeste. Brasília: Ministério do Meio Ambiente; 2018. 19 p.

2. Silva ACC, Silva NA, Pereira MCS, Vassimon HS. Alimentos contendo ingredientes funcionais em sua formulação: revisão de artigos publicados em revistas brasileiras. Rev Con Ci Onl. 2016 Dec;11(2):133144, doi: $10.24862 /$ cco.v11i2.429.

3. Santos OV, Correa NCF, Junior RNC, Costa CEF, Moraes JFC, Lannes SCS. Quality parameters and thermogravimetric and oxidative profile of muruci oil (Byrsonima crassifolia L.) obtained by supercritical CO2. Food Sci Technol. 2018 Jan;38(1):172-9, doi: 10.1590/ 1678-457x.30616.

4. Moreira-Araújo, RSR, Barros NVA, Porto RGCL, Brandão ACAS, Fett R. Bioactive compounds and antioxidant activity three fruit species from the Brazilian Cerrado. Rev Bras Frutic. 2019 Jun;41(3):e011, doi: 10.1590/0100-29452019011.

5. Costa RS, Santos OV, Lannes SCS, Casazza AA, Aliakbarian B, Perego, Ribeiro-Costa RM, Coverti A, Silva-Junior JOC. Bioactive compounds and value-added applications of cupuaçu (Theobroma grandiflorum Schum.) agroindustrial by-product. Food Sci Technol. 2019 Apr,40(2):401-7, doi: 10.1590/ 1678-457x.30616.

6. Nascimento LM, Gomes KRO, Mascarenhas MDM, Miranda CES, Araújo TME, Frota KMG. Association between the consumption of antioxidant nutrients with lipid alterations and cardiometabolic risk in adolescents. Rev Nutr. 2018 Mar;31(2):183-97 doi: 10.1590/1678-98652018000200005.

7. Morais MF, Santos JR, Santos MP, Santos DC, Costa TN, Lima JB. Modeling and thermodynamic properties of bacaba pulp drying. Rev Bras Eng Agríc Ambient. 2019 Jul;23(9):702-8, doi: 10.1590/1807-1929.

8. Vieira LM. Caracterização química e capacidade antioxidante in vitro do coco babaçu (Orbignya speciosa) [dissertação]. Teresina (PI): Universidade Federal do Piauí; 2011. 93 p.

9. AOAC. Association of Official Analytical Chemistry. Official Methods of Analysis of the Association of Official Analytical Chemistry. 18 ${ }^{\text {th }}$ ed. Washington (DC): Gaithersburg; 2010. 1094 p.

10. IAL. Instituto Adolfo Lutz. Normas analíticas do Instituto Adolfo Lutz: métodos químicos e físicos para análise de alimentos. São Paulo: IMESP; 1985. 533 p.

11. IAL. Instituto Adolfo Lutz. Métodos físico-químicos para análise de alimentos. São Paulo: Instituto Adolfo Lutz; 2008. 1020 p.

12. Rodriguez-Amaya DB. A guide to carotenoid analysis in foods. Washington: ILSI Press; 2001. 64 p.

13. Lees DH, Francis FJ. Standardization of pigment analyses in cranberries. Hortscience. 1972;7(1):83-4. 
14. Alikbarian B, Casazza AA, Perego P. Valorization of olive oil solid waste using high pressure-high temperature reactor. Food Chem. 2011 May;128(3):704-10, doi: 10.1016/j.foodchem.2011.03.092.

15. Carvalho AV, do Nascimento WMO. Caracterização físico-química e química da polpa de frutos de muruci. Boletim de Pesquisa e Desenvolvimento 108. Belém: Embrapa Amazônia Oriental; 2016. 17 p.

16. Monteiro DCB, Pires CRF. Avaliação da estabilidade físico-química de geléias de murici armazenadas sob diferentes condições de temperatura e luminosidade. DES-UFT. 2016;(3):87-98, doi: 10.20873/uft.2359-3652.2016v3nespp87.

17. da Conceição Souza JLC, Silva LB, Reges NPR, Mota EES, Leonidio RL. Caracterização física e química de gabiroba e murici. Rev Cienc Agrar. 2019 Sep;42(3):792-800, doi: 10.19085/rca.17521.

18. López E, Navarro A, Manchhon N, Herrera J. Componentes funcionales en Manche (Byrsonimia crassifolia (L) Kunth). Cuerp Académi. 2014 Sep;2:6-22.

19. Alves VM, Silva EP, Silva AGM, Asquieri ER, Damiani C. Gabiroba and murici: study of the nutritional and antinutritional value of peel, pulp and seed. Res Soc Dev. 2020 Mar;9(5):e152953260, doi: $10.33448 /$ rsd-v9i5.3260.

20. Brasil. Ministério da Agricultura, Pecuária e Abastecimento. Portaria no 58, de 30 de agosto de 2016. Estabelece em todo território nacional a complementação dos padrões de identidade e qualidade de polpa de fruta. Brasília (DF): Diário Oficial da União, ed. 169; 2016. p. 2.

21. Brasil. Ministério da Agricultura, Pecuária e Abastecimento. Instrução Normativa $\mathrm{N}^{\circ} 37$, de $1^{\circ}$ de outubro de 2018. Parâmetros analíticos e quesitos complementares aos padrões de identidade e qualidade de polpa de fruta. Brasília (DF): Diário Oficial da União, ed. 194; 2018. p. 28.

22. Brasil. Agência Nacional de Vigilância Sanitária. Guia para determinação de prazos de validade de alimentos. Anvisa. 2018 Out;16(1):1-76.

23. Maisnam D, Rasane P, Dey A, Kaur S, Sarma C. Recent advances in conventional drying of foods. J Food Technol Pres. 2017 Fev;1(1):25-34.

24. Mota VA, Castro JC, Vagula JM, Costa JMC, Clemente E. Physicochemical quality of murici covered with starch based coverings and stored at different temperatures. Afr J Agric Res. 2016 April;11(15):1344-52, doi: 10.5897/AJAR2015.9723.

25. Peralta MÁM, Santillán PS, García ARR, Lagarda JLV, Luzardo MBB, Luis Alaniz Gutiérrez LA. Caracterización y evaluación de frutos de 'nanche' (Byrsonima crassifolia L.). Rev Mex Cienc Agríc. 2020 Fev;11(1):151-160, doi: 10.29312/remexca.v11i1.1950

26. Santos OV, Soares SD, Vieira ELS, Lisboa LRC, Pinto DML, Maciel ACC, Nascimento FCA. Excessive Consumption of Potentially Erosive beverages: implications for publichealth. Braz J Hea. $2019 \mathrm{Jul} /$ Aug;2(40):2554-2571, doi: 10.34119/bjhrv2n4-027.

27. Carlos ND, Loss RA, Silva SS, Guedes SF, Carvalho JWP. Avaliação físico-química e atividade antimicrobiana da casca, polpa e semente do murici (Byrsonima crassifolia). Encicl Biosf. 2017 Jun;14(25):232-43, doi: 10.18677/EnciBio_2017A22.

28. Santos EHF, Figueiredo Neto A, Donzeli VP. Aspectos físico-químicos e microbiológicos de polpas de frutas comercializadas em Petrolina (PE) e Juazeiro (BA). Braz J Food Technol. 2016 Dez;19:1-9, doi: 10.1590/1981-6723.8915.

29. Brasil. RDC n 269 de 22 de setembro de 2005. Aprova o regulamento técnico sobre a ingestão diária recomendada (IDR) de proteína, vitaminas e minerais. Ministério da Saúde - MS. Agência Nacional de Vigilância Sanitária - Anvisa [Internet]. [place unknown]: Anvisa; 2005. [citado em 21 de junho de 2020]. 6 p. Disponível em: https://coffito.gov.br/nsite/wp-content/uploads/2016/08/resoluo-rdc-n-2692005-ingesto-diria-recomendada-idr-de-protenas-vitaminas-e-minerais.pdf.

30. Belisario CM, Soares AG, Coneglian RCC, Placido GR, Castro CFS, Rodrigues LAN. Carotenoids, sugars, ascorbic acid, total phenolics, and antioxidant activity of murici from Brazilian Cerrado during refrigerated storage. Cienc Rur. 2020 Mar;50 (4):e20180620, doi: 10.1590/0103-8478cr20180620.

31. Franklin B, Nascimento FCA. Plantas para o futuro: compilação de dados de composição nutricional do araçá-boi, buriti, cupuaçu, murici e pupunha. Braz J Develop. 2020 Mar;6(3):10174-189, doi: $10.34117 / \mathrm{bjdv}$

32. Tabela Brasileira de Cmposição de Alimentos (TBCA). Tabelas complementares - Vitamina A e carotenoides. Universidade de São Paulo (USP). Food Research Center (FoRC). Versão 7.0 [Internet]. [São Paulo]: OBTZ-Tech; 2019 [citado em 2019]. Disponível em: http://www.fcf.usp.br/tbca.

33. Britton G, Khachik F. Carotenoids in food. In: Britton G, Pfander H, Liaaen-Jensen S, editors. Carotenoids. Vol. 5, Carotenoids. [place unknown]: Birkhäuser Basel; 2009. p. 45-66, doi: 10.1007/9783-7643-7501-0_3.

34. Bohn T. Carotenoids and markers of oxidative stress in human observational studies and intervention trials: Implications for chronic diseases. Antioxid. 2019 Jun;8(6):1-44, doi: 10.3390/antiox8060179.

35. Tan BL, Norhaizan ME. Carotenoids: How effective are they to prevent age-related diseases? Molecul. 2019 May;24(9):1-23, doi: 10.3390/molecules24091801. 
36. Araujo ACMA, Menezes EGT, Terra AWC, Dias BO, Oliveira ER, Queiroz F. Bioactive compounds and chemical composition of Brazilian Cerrado fruits' wastes: pequi almonds, murici, and sweet passion fruit seeds. Food SciTechnol. 2018 Dec;38(2):203-14, doi: 10.1590/fst.19417.

37. Awuchi CG, Igwe SV, Amagwula I, Kate EC. Health benefits of micronutrients (vitamins and minerals) and their Associated Deficiency Diseases: a systematic review. Int J Food Sci. 2020;3(1):1-32.

38. Ramalho A. Vitamina A. Série de publicações ILSI Brasil: funções plenamente reconhecidas de nutrientes. 2. ed. São Paulo: ILSI Brasil - International Life Sciences Institute; 2010.

39. NHI. National Institutes of Health. Office of Dietary Supplements. Vitamin A. Fact Sheet for Health Professionals [Internet]; 14 Fev 2020. Disponível em: https://ods.od.nih.gov/factsheets/VitaminAHealthProfessional/ [Acesso em 05 Dez 2020].

40. Becker MM, Mandaji CM, Catanante G, Marty JL, Nunes GS. Mineral and bromatological assessment and determination of the antioxidant capacity and bioactive compounds in native Amazon fruits. Braz J Food Technol. 2018 Aug.;21(16):1-9, doi: 10.1590/1981-6723.02218.

41. Santos MFG, Mamede RVS, Rufino MSM, Brito ES, Alves RE. Amazonian native palm fruits as sources of antioxidant bioactive compounds. Antioxidants. 2015 Set;4(1):591-602, doi: $0.3390 /$ antiox 4030591 .

42. Gonçalves NP, Lucena EMP, Bonilla OH, Silveira MRS, Tavares FJC. Bioactive compounds during the maturation of four fruits native to the restinga forest of Ceará. Rev Bras Frutic. 2017 Dec;39(5):110, doi: 10.1590/0100-29452017686

43. Montenegro J, Aniceto A, De Abreu JP, Teodoro AJ. Características físico-químicas e atividade antioxidante de frutas da região Amazônica. In: Anais da $69^{\mathrm{a}}$ Reunião Anual da SBPC; 16 a 22 de julho de 2017; Belo Horizonte, MG. [Minas Gerais]: UFMG; 2017. 4 p.

44. Andrade RSG, Diniz MCT, Neves EA, Nobrega JA. Determinação e distribuição de ácido ascórbico em três frutos tropicais. Eclética Quím J. 2002 Dec;27:393-401, doi: 10.1590/S0100-46702002000200032

45. Kamiloglu S, Toydemir G, Boyacioglu D, Beekwilder J, Hall RD, Capanoglu E. A review on the effect of drying on antioxidant potential of fruits and vegetables. Crit Rev Food Sci Nutr. 2016 Jul;56(1):S110S129, doi: $10.1080 / 10408398.2015 .1045969$

46. Amit R, Bikash M, Ravindra B. Supercritical extraction of sunflower oil: A central composite design for extraction variables. Food Chem. 2016;192:647-659, doi: 10.1016/j.foodchem.2015.07.070

47. Santos OV, Carvalho RN, Costa CEF, Lannes SCS. Chemical, chromatographic-functional, thermogravimetric-differential and spectroscopic parameters of the sapucaia oil obtained by different extraction methods. J Ind Crops. 2019;132:487-496, doi: 10.1016/j.indcrop.2019.02.043.

48. Se KW, Ghoshal SK, Wahab RA, Ibrahim RKI, Lani MN. A simple approach for rapid detection and quantification of adulterants in stingless bees (Heterotrigona itama) honey. Food Res Int. 2018;105:453-460, doi: 10.1016/j.foodres.2017.11.012. 